\title{
Newer isn't always better: comparative effectiveness of sevoflurane and isoflurane in cardiac surgery
}

\author{
Michael R. Mathis, MD (1) - Sachin Kheterpal, MD, MBA
}

Received: 25 June 2016/Revised: 3 July 2016/Accepted: 14 July 2016/Published online: 28 July 2016

(c) Canadian Anesthesiologists' Society 2016

In this issue of the Journal, Jones et al. ${ }^{1}$ present a comparative effectiveness trial showing no difference in clinically relevant outcomes between sevoflurane and isoflurane when used for anesthetic maintenance during cardiac surgery. Specifically, the authors showed that sevoflurane is non-inferior to isoflurane based on a primary outcome composite measure of prolonged intensive care unit (ICU) length of stay (LOS) and all-cause 30-day mortality. Furthermore, the authors found that sevoflurane is not superior to isoflurane on any other clinically important outcome.

The direct ramifications of this study are interesting. For instance, the authors suggest potentially saving millions of healthcare dollars per year if anesthesiologists opt to use isoflurane rather than sevoflurane during cardiac surgery. Of greater interest, however, is the fact that this study was just now performed, despite both anesthetics being available for over 20 years in the context of clinical equipoise. This work highlights that scientific research should not only strive to push the bounds of basic knowledge but also welcome pragmatic studies performed in real-world settings to address daily clinical conundrums.

Consider recent research in our field comparing isoflurane with sevoflurane. This work includes a study in a rodent model assessing electroencephalographic activity ${ }^{2}$ as well as a study assessing aminotransferase levels during hepatic ischemia-reperfusion injury ${ }^{3}$ in a similar animal model. Although this type of comparative basic science

M. R. Mathis, MD $(\varangle) \cdot$ S. Kheterpal, MD, MBA

Department of Anesthesiology, University of Michigan Health System, 1H247 UH, SPC 5048, 1500 East Medical Center Drive, Ann Arbor, MI 48109-5048, USA

e-mail: mathism@med.umich.edu research yields valuable and often mechanistic findings, which should be commended and selectively encouraged, it should not be at the exclusion of pragmatic clinical trials such as that performed by Jones et al. Their comparative effectiveness study achieves its value by answering a common clinical question with an easily graspable healthcare impact, all the while engaging patients as participants in the progress of science. Indeed, the value of these trials has been previously shown and promoted among healthcare policy-makers who esteem "the art of the possible". 4 To continue increasing its impact on science, the field of anesthesiology must complement and expand on its historical strengths in basic science and patient safety research with the expansion of comparative effectiveness research.

Beyond an admirably practical study design, the work of Jones et al. sheds new light on a topic particularly germane to cardiac anesthesia. Among studies of volatile anesthetics used in cardiac surgery, meta-analyses have previously shown cardioprotective effects of volatile agents when compared with intravenous anesthetics. ${ }^{5,6}$ Comparing the differential effects of volatile anesthetics, studies have shown relatively less depressed systolic and diastolic function and smaller increases in heart rate in subjects receiving sevoflurane compared with isoflurane; however, these studies were limited by a lack of long-term outcomes and real-world applicability. ${ }^{7,8}$ Although these prior studies suggest that sevoflurane may provide a smoother set of hemodynamic dots and dashes on an anesthetic record, the impact this may have on any meaningful measure of healthcare is less apparent. Like many areas currently in medicine, this knowledge gap can often be bridged most effectively by a pragmatic trial. Through their work, Jones et al. make progress in rigorously evaluating (and dispelling) perceived differences between sevoflurane and 
isoflurane, within the context of healthcare outcomes arguably more relevant to the broader medical community.

Nevertheless, advances made through comparative effectiveness trials, such as that by Jones et al., should not minimize scientific progress achieved through other research techniques. Although previously underutilized, comparative effectiveness trials represent just one tool within the healthcare researcher's armamentarium, and they are not without their own limitations. Indeed, one such limitation to studies performed using pragmatic trial design-defined as a design to help choose between routine options for care $^{9}$ - is ironically that they are actually "pragmatic". While pragmatic trials may realize practical solutions, these studies often offer no greater mechanistic understanding of healthcare interventions. In this present study, the authors provide clinical evidence to anesthesiologists deliberating between choosing either isoflurane or sevoflurane for cardiac surgery. Nevertheless, the aforementioned limitation is that the trial offers little insight into the pathophysiologic mechanisms of sevoflurane $v s$ isoflurane that might contribute to their similar outcomes, despite previous demonstrable differences in hemodynamic effects.

Furthermore, whereas large pragmatic trials may best elaborate on the progress made in research through small incremental changes, progress requiring a larger paradigm shift may not be possible with such an approach. While the authors make a case for isoflurane as a cost-effective anesthetic when compared with sevoflurane for cardiac surgery, this perspective is limited to the range of possibilities that are not only known but also readily available. Consider notable recent advances in cardiac surgery, including the development of transcatheter valve implantation, ${ }^{10}$ innovations in transesophageal echocardiography, ${ }^{11}$ and the development of short-acting anesthetics promoting ICU fast-tracking. ${ }^{12}$ In each case, the presiding healthcare model changed dramatically in order to achieve benefit-similar development would not likely have been feasible through pragmatic trials.

Apart from discussions of study design and comparative effectiveness, Jones et al. invoke a discussion of healthcare cost in their study. This is a regular feature of noninferiority designs-i.e., when the therapeutics are similar in effectiveness (i.e., non-inferior to one another), then the choice as to which one to use often falls on price. Indeed, the authors conclude that, among outcomes appreciated by healthcare policymakers, perhaps the most striking difference between sevoflurane and isoflurane has nothing to do with preserved hemodynamics or myocardial preservation (much to the anesthesiologist's disappointment); rather, it has more to do with the cost of each drug. The authors' efforts are laudable for encouraging a cost-conscious approach to medical decision-making. Regarding true cost differentials between volatile agents, however, perhaps a more thorough cost-effectiveness analysis is justified. For example, could costs be reduced by reducing fresh gas flows, or could one volatile agent require use of more healthcare resources (i.e., laboratory data, medication coadministration, and postoperative care)? It is worth highlighting a trend towards significance favouring sevoflurane in reducing postoperative ICU LOS. Even a small difference in effect size-which may require a greater study power for detection-would translate to ICU costs likely far eclipsing any cost saved with a less expensive volatile anesthetic. While Jones et al. succeed in designing a study comparing sevoflurane with isoflurane among clinical measures, several economic questions remain unanswered. These questions of cost justify a comparative efficiency study, designed to assess meaningful differences in cost, rather than clinical effect, between sevoflurane and isoflurane during cardiac surgery.

Unanswered questions of cost and pathophysiology aside, the potential impact of the work by Jones et al. should not be underappreciated. Through their pragmatically designed study, the authors humbly reach a conclusion somewhat against prevailing practice. As supported by this study, a cardiac anesthesiologist may now comfortably opt to use isoflurane rather than sevoflurane, given a lack of effect on clinically meaningful outcomes. In a world where healthcare providers' conviction to certain clinical practice methods are often disproportionate to the strength of existing clinical evidence, a focus on getting the "simple" details right—as Jones et al. set out to do-becomes justified.

In conclusion, the scientific merit of the work of Jones et al. is evident and represents an impactful definitive step forward for cardiac anesthesia practice. By leveraging a practical approach to studying volatile anesthetics, the authors address a critical knowledge gap and open the door for thoughtful discussion of cost-effectiveness to drive decision-making for patients receiving volatile anesthetics for cardiac surgery.

\section{Nouveauté ne rime pas toujours avec progrès: l'efficacité comparative du sévoflurane et de l'isoflurane en chirurgie cardiaque}

Dans ce numéro du Journal, Jones et coll. ${ }^{1}$ présentent une étude d'efficacité comparative qui ne montre aucune différence clinique significative entre le sévoflurane et 
l'isoflurane lorsque ces agents sont utilisés pour le maintien de l'anesthésie pendant une chirurgie cardiaque. Plus spécifiquement, les auteurs ont démontré que le sévoflurane était non inférieur à l'isoflurane selon une mesure composite du critère d'évaluation principal, mesure qui comprenait une durée de séjour prolongée à l'unité de soins intensifs (USI) et la mortalité, toutes causes confondues, à 30 jours. Qui plus est, les auteurs ont découvert que le sévoflurane n'était pas supérieur à l'isoflurane en ce qui touchait à n'importe quel autre critère clinique important.

Les ramifications directes de cette étude sont intéressantes. Par exemple, les auteurs suggèrent une économie potentielle de millions de dollars par année dans les soins de santé si les anesthésiologistes décidaient d'utiliser l'isoflurane plutôt que le sévoflurane pendant les chirurgies cardiaques. Ce qui est encore plus intéressant toutefois, c'est que l'étude n'a été réalisée qu'aujourd'hui, alors que ces deux agents sont disponibles depuis plus de 20 ans dans un contexte d'incertitude clinique absolue. Ces travaux soulignent le fait que la recherche scientifique devrait non seulement s'efforcer de repousser les limites des connaissances de base, mais également accueillir les études pragmatiques réalisées dans le monde réel et s'intéressant aux mystères cliniques de tous les jours.

Pensez aux recherches récentes menées dans notre domaine et comparant l'isoflurane au sévoflurane. Parmi ces travaux, citons une étude portant sur un modèle de rongeurs évaluant l'activité électro-encéphalographique ${ }^{2}$ ainsi qu'une étude évaluant les taux d'aminotransférase observés pendant une lésion d'ischémie-reperfusion hépatique $^{3}$ chez un modèle animal semblable. Bien que ce type de recherches comparatives en science fondamentale donne souvent des résultats précieux et mécanistes-des efforts louables et à encourager dans une certaine mesure-, ces travaux ne devraient pas être réalisés à l'exclusion d'études cliniques pragmatiques telles que celle de Jones et coll. La valeur de leur étude d'efficacité comparative réside en ce qu'elle répond à une question clinique fréquente ayant un impact facile à comprendre sur les soins de santé, tout en engageant les patients comme participants aux progrès de la science. En effet, la valeur de telles études a déjà été démontrée et promue parmi les décideurs en santé qui admirent « l'art du possible $»{ }^{4}$ Afin de continuer à augmenter son impact sur la science, le domaine de l'anesthésiologie doit complémenter sa force traditionnelle en science fondamentale et en recherche sur la sécurité des patients, par une expansion de la recherche en efficacité comparative.

Outre une méthodologie d'étude fort pratique, les travaux de Jones et coll. jettent une lumière nouvelle sur un sujet particulièrement pertinent à l'anesthésie cardiaque.
Parmi les études portant sur les agents volatils utilisés en chirurgie cardiaque, des méta-analyses ont démontré les effets cardioprotecteurs de ces agents par rapport aux agents anesthésiques intraveineux. ${ }^{5,6}$ En comparant les effets différentiels des anesthésiques volatils, des études ont montré une baisse relativement moins importante des fonctions systolique et diastolique et de plus petites augmentations de la fréquence cardiaque chez les patients recevant du sévoflurane par rapport à ceux recevant de l'isoflurane; toutefois, ces études étaient limitées par l'absence d'issues à long terme et d'applicabilité concrète. ${ }^{7,8}$ Bien que, selon ces études antérieures, le sévoflurane procurerait un tracé plus linéaire de points et de traits hémodynamiques dans le dossier anesthésique, l'impact que cela pourrait avoir sur quelque mesure d'importance en matière de soins de santé est moins facile à démontrer. Comme dans bon nombre de domaines en médecine aujourd'hui, ce manque de connaissances peut souvent être aisément pallié par une étude pragmatique. Grâce à leurs travaux, Jones et coll. vont de l'avant en évaluant de façon rigoureuse (et en écartant) les différences perçues entre le sévoflurane et l'isoflurane, dans le contexte de critères d'évaluation de soins de santé probablement plus pertinents à la communauté médicale dans son ensemble.

Cependant, les progrès réalisés grâce à des études d'efficacité comparative, telles que celle de Jones et coll., ne devraient pas minimiser les progrès scientifiques enregistrés grâce à d'autres techniques de recherche. Bien que précédemment sous-utilisées, les études d'efficacité comparative ne sont qu'un outil de l'arsenal du chercheur en soins de santé, et elles comportent elles aussi leurs limites. En fait, l'une des limites des études réalisées à l'aide d'une méthodologie pragmatique-définie en tant que méthodologie visant à éclairer le choix entre diverses options de soins ${ }^{9}$ répandues-est, de façon tout à fait ironique, qu'elles sont en fait «pragmatiques ». Bien que les études pragmatiques puissent offrir des solutions pratiques, elles ne proposent bien souvent aucun approfondissement de notre compréhension mécaniste des interventions de soins de santé. Dans l'étude présentée ici, les auteurs présentent des données cliniques factuelles aux anesthésiologistes se demandant quelle est la meilleure option pour la chirurgie cardiaque, soit l'isoflurane ou le sévoflurane. Toutefois, la limite mentionnée ci-dessus réside dans le fait que cette étude n'offre qu'une perspective limitée des mécanismes physiopathologiques du sévoflurane $v s$ l'isoflurane qui pourraient contribuer à leurs issues semblables, malgré les différences démontrables dans leurs effets hémodynamiques.

De plus, alors que les études pragmatiques d'envergure sont les mieux à même d'approfondir les progrès de la recherche en appliquant de petits changements progressifs, 
les progrès nécessitant un transfert de paradigme plus important pourraient ne pas être possibles avec une telle approche. Alors que les auteurs préconisent l'isoflurane en tant qu'anesthésique rentable plutôt que le sévoflurane en chirurgie cardiaque, cette perspective se limite à l'éventail de possibilités qui sont non seulement connues, mais aussi facilement disponibles. Pensez aux importants progrès récents en chirurgie cardiaque, notamment la mise au point de l'implantation valvulaire transcathéter, ${ }^{10}$ les innovations en échocardiographie transœsophagienne, ${ }^{11}$ et la mise au point d'agents anesthésiques à action brève pour favoriser l'extubation rapide à l'USI. ${ }^{12}$ Dans chacun de ces cas, le modèle de soins de santé en place a dû subir de profonds changements pour pouvoir profiter des avantages; un développement semblable n'aurait probablement pas été possible si l'on était passé par des études pragmatiques.

Outre leurs discussions concernant la méthodologie d'étude et l'efficacité comparative, Jones et coll. abordent le sujet des coûts des soins de santé dans leur étude. Il s'agit d'une caractéristique fréquente des études de non-infériorité-en d'autres mots, lorsque les traitements sont semblables en terme d'efficacité (c.-à-d. non inférieurs l'un à l'autre), alors le choix quant à quel traitement utiliser se rabat souvent sur leur prix. En effet, les auteurs concluent que, parmi les critères d'évaluation appréciés par les décideurs en soins de santé, la différence la plus frappante entre le sévoflurane et l'isoflurane n'a peut-être rien à voir avec la préservation de l'hémodynamie ou du myocarde (au grand dam des anesthésiologistes); plutôt, elle se fonde davantage sur le coût de chaque médicament. Les efforts des auteurs sont louables en ce qu'ils encouragent une approche de la prise de décision médicale qui tienne compte des coûts. En ce qui touche aux véritables différentiels de coûts entre les agents volatils, toutefois, une analyse de coût-efficacité plus approfondie est peut-être justifiée. Par exemple, peut-on réduire les coûts en réduisant les débits de gaz frais, ou serait-il possible qu'un agent volatil nécessite l'utilisation de davantage de ressources en santé (par ex., les données de laboratoire, la co-administration de médicaments, et les soins postopératoires)? Il est important de souligner une tendance quasi significative selon laquelle le sévoflurane réduirait la durée de séjour à l'USI en période postopératoire. Même une petite différence de la taille de l'effet-qui pourrait nécessiter une plus grande puissance d'étude pour être détectée—se traduirait en coûts de l'USI éclipsant probablement de loin toute économie réalisée en choisissant un anesthésique volatil moins dispendieux. Bien que Jones et coll. aient réussi à mettre au point une étude comparant le sévoflurane et l'isoflurane parmi des mesures cliniques, plusieurs questions d'ordre économique demeurent sans réponse. Ces questions de coût justifient une étude d'efficacité comparative, conçue pour évaluer les différences significatives en matière de coût, plutôt que d'effet clinique, entre le sévoflurane et l'isoflurane pendant une chirurgie cardiaque.

Hormis les questions de coûts et de physiopathologie restées sans réponse, l'impact potentiel des travaux de Jones et coll. ne devrait pas être sous-estimé. Grâce à leur étude pragmatique, les auteurs aboutissent à une conclusion qui va quelque peu à contre courant de la pratique dominante. Comme cette étude le soutient, un anesthésiologiste cardiaque peut désormais décider en toute tranquillité d'utiliser de l'isoflurane plutôt que du sévoflurane, étant donné l'absence d'effets sur les critères d'évaluation clinique. Dans un monde où les convictions des professionnels de la santé concernant certaines méthodes de pratique clinique sont souvent disproportionnées par rapport aux données cliniques existantes, il devient justifié de s'appliquer à faire correctement les choses simples, et c'est là la mission que Jones et coll. s'étaient fixée.

Pour conclure, le mérite scientifique des travaux de Jones et coll. est évident et représente un pas décisif en avant pour la pratique de l'anesthésie cardiaque. En s'appuyant sur une approche pratique de l'étude des agents volatils, les auteurs s'intéressent à une lacune cruciale et ouvrent la porte à des discussions réfléchies de la rentabilité comme moteur de prise de décision pour les patients recevant des anesthésiques volatils pour une chirurgie cardiaque.

Funding All work and partial funding attributed to the Department of Anesthesiology, University of Michigan Medical School. The project described was supported in part by the National Center for Research Resources, Grant UL1RR024986, which is now at the National Center for Advancing Translational Sciences, Grant 2UL1TR000433. The content is solely the responsibility of the authors and does not necessarily represent the official views of the National Institutes of Health.

Conflicts of interest None declared.

Editorial responsibility This submission was handled by Dr. Hilary P. Grocott, Editor-in-Chief, Canadian Journal of Anesthesia.

Financement Tous les travaux et un financement partiel sont attribués au département d'anesthésiologie de l'École de médecine de l'Université du Michigan. Le projet décrit a été financé en partie par le National Center for Research Resources, Bourse UL1RR024986, qui est aujourd'hui au National Center for Advancing Translational Sciences, Bourse 2UL1TR000433. Ce contenu reflète exclusivement la responsabilité des auteurs et ne représente pas nécessairement le point de vue officiel des National Institutes of Health.

\section{Conflit d'intérêt Aucun.}

Responsabilité éditoriale Cet article a été traité par Dr Hilary P. Grocott, rédacteur en chef, Journal canadien d'anesthésie. 


\section{References}

1. Jones PM. Bainbridge D, Chu MW, et al. Comparison of isoflurane and sevoflurane in cardiac surgery: a randomized noninferiority comparative effectiveness trial. Can J Anesth 2016; 63: this issue. DOI: 10.1007/s12630-016-0706-y

2. Seubert CN, Zhu W, Pavlinec C, Gravenstein N, Martynyuk AE. Developmental effects of neonatal isoflurane and sevoflurane exposure in rats. Anesthesiology 2013; 119: 358-64.

3. Bedirli N, Ofluoglu E, Kerem $M$, et al. Hepatic energy metabolism and the differential protective effects of sevoflurane and isoflurane anesthesia in a rat hepatic ischemia-reperfusion injury model. Anesth Analg 2008; 106: 830-7.

4. Maclure M. Explaining pragmatic trials to pragmatic policymakers. CMAJ 2009; 180: 1001-3.

5. Landoni G, Biondi-Zoccai GG, Zangrillo A, et al. Desflurane and sevoflurane in cardiac surgery: a meta-analysis of randomized clinical trials. J Cardiothorac Vasc Anesth 2007; 21: 502-11.

6. Yи $\mathrm{CH}$, Beattie WS. The effects of volatile anesthetics on cardiac ischemic complications and mortality in CABG: a meta-analysis. Can J Anesth 2006; 53: 906-18.
7. Skeehan TM, Schuler HG, Riley JL. Comparison of the alteration of cardiac function by sevoflurane, isoflurane, and halothane in the isolated working rat heart. J Cardiothorac Vasc Anesth 1995; 9: 706-12.

8. Nishiyama T. Hemodynamic and catecholamine response to a rapid increase in isoflurane or sevoflurane concentration during a maintenance phase of anesthesia in humans. J Anesth 2005; 19: 213-7.

9. Zwarenstein $M$, Treweek $S$, Gagnier JJ, et al. Improving the reporting of pragmatic trials: an extension of the CONSORT statement. BMJ 2008; 337: a2390.

10. Myat A, Hildick-Smith D, Young C, Thomas M, Redwood SR. Transcatheter aortic valve implantation: revolution and evolution 10 years on. Heart 2012; 98 Suppl 4: iv1-6.

11. Smith WB, Robinson AR 3rd, Janelle GM. Expanding role of perioperative transesophageal echocardiography in the general anesthesia practice and residency training in the USA. Curr Opin Anaesthesiol 2015; 28: 95-100.

12. Lena P, Balarac $N$, Lena $D$, et al. Fast-track anesthesia with remifentanil and spinal analgesia for cardiac surgery: the effect on pain control and quality of recovery. J Cardiothorac Vasc Anesth 2008; 22: 536-42. 